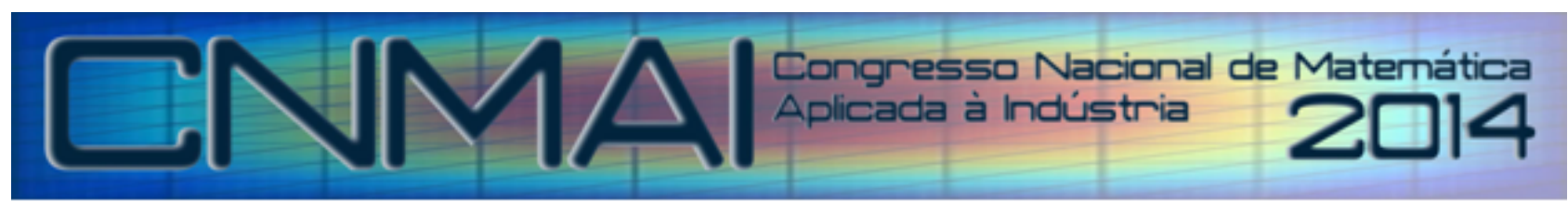

18 a 21 de novembro de 2014, Caldas Novas - Goiás

\title{
DESENVOLVIMENTO DE UMA METODOLOGIA PARA O TRATAMENTO DE CARCINOMAS ATRAVÉS DA RESOLUÇÃO DE PROBLEMAS INVERSOS
}

\author{
Fran Sérgio Lobato, fslobato@feq.ufu.br ${ }^{3}$ \\ ${ }^{1}$ Faculdade de Engenharia Química, Universidade Federal de Uberlândia, Brasil \\ Av. João Naves de Ávila, 2.121 - Bloco 1K, 38408-100, Uberlândia - MG
}

\begin{abstract}
Resumo. A análise de problemas de engenharia via formulação e resolução de problemas diretos e inversos constitui uma área da ciência de grande aplicabilidade nos dias atuais. Esse interesse se deve aos modelos matemáticos cada vez mais realísticos associados aos sofisticados métodos numéricos empregados para a sua simulação. Neste cenário, a aplicação de análise inversa para a determinação de regiões que apresentam células cancerígenas constitui uma tarefa de grande relevância, já que um dos tipos de tratamentos existentes consiste em bombardear estas regiões com radioterapia. Diante do que foi apresentado, o presente trabalho tem por objetivo determinar regiões que apresentam carcinomas de pele através da formulação e resolução de um problema inverso via modelagem matemática do processo de transferência de calor em tecidos associado ao Algoritmo de Evolução Diferencial. Os resultados preliminares indicam que metodologia proposta configura-se como uma interessante alternativa para esta finalidade.
\end{abstract}

Palavras-chave: Transferência de Calor em Tecidos, Problema Inverso, Evolução Diferencial.

\section{INTRODUÇÃO}

Atualmente, o desenvolvimento de novas estratégias para o tratamento de doenças do nosso cotidiano é tema de pesquisas no mundo todo. Neste cenário, o uso de modelos matemáticos para a caracterização da evolução de tais doenças e, consequentemente, o estudo de estratégias para o tratamento das mesmas, configura-se como uma área de grande importância entre matemáticos e engenheiros. Matematicamente, a modelagem destes fenômenos se dá por meio de equações algébrico-diferenciais que representam os balanços de massa, energia e quantidade de movimento.

Dentre as inúmeras aplicações desenvolvidas atualmente, o estudo de novas estratégias para o tratamento de carcinomas representa um tema relevante atualmente (Agnelli et al., 2011; Lima et al., 2006). Segundo dados do Instituto Nacional do Câncer (INCA), a ocorrência de câncer em território brasileiro será de aproximadamente 576 mil casos novos em 2014. Dentre os tipos de câncer, o de pele do tipo não melanoma será o mais incidente na população brasileira, com estimativa prevista de 182 mil casos novos ${ }^{1}$.

Apesar de sua baixa letalidade em relação a outros tipos de tumores malignos, o câncer de pele é o tipo mais prevalente na população humana, sendo desta forma considerado um problema de saúde pública no mundo. O câncer de pele é provocado pelo crescimento anormal e descontrolado das células que compõem a pele. Estas células se dispõem formando camadas e, de acordo com a camada afetada, são definidos os diferentes tipos de câncer. O seu surgimento está diretamente relacionado à exposição solar exagerada, ao uso de câmaras de bronzeamento artificial, à tonalidade da pele, ao avanço da idade da população e apresenta um padrão de distribuição geográfica. O câncer de pele é dividido em dois grandes grupos: o melanoma e o não melanoma. O primeiro é considerado uma entidade à parte, tem uma baixa incidência (cerca de 3 casos por 100.000 pessoas/ano) e história natural bastante diferente, sendo mais agressivo, de crescimento rápido e com maior potencial de disseminação para outros órgãos. Já os tumores de pele não melanomas são doenças muito mais comuns (cerca de 70 casos por 100.000 habitantes/ano), possuem um curso clínico mais indolente, lento crescimento e raramente disseminam para órgãos a distância (2 a $5 \%$ dos casos) ${ }^{2}$.

\footnotetext{
${ }^{1}$ http://saudeweb.com.br/

${ }^{2} \mathrm{http}: / /$ www.sbd.org.br/
} 
O tratamento deste tipo de câncer depende de uma série de fatores, dentre os quais pode-se citar: idade, localização e profundidade da lesão, questões estéticas e econômicas. Normalmente é feita a retirada cirúrgica da lesão, mas a crioterapia (congelação) ou eletrocauterização (queimadura) podem sem indicadas em casos selecionados. Além das modalidades citadas, a radioterapia, a quimioterapia, a imunoterapia e as medicações orais e tópicas são outras opções de tratamento para os carcinomas ${ }^{3}$.

Para o caso específico da radioterapia, onde a radiação ionizante (processo de produção química com espécies eletricamente carregadas, através da perda ou ganho de elétrons, atuando sobre moléculas neutras ou átomos) é empregado para destruir, eliminar ou impedir que as células de um tumor aumentem seu tamanho, é de extrema importância que o tumor (ou a região) seja localizado. Conforme citado anteriormente, o sistema termo-regulatório humano tem a capacidade de manter a temperatura média interna em torno de $37^{\circ} \mathrm{C}$ sob uma ampla faixa de condições ambientais externas e durante tensões térmicas (Pennes, 1948). Neste contexto, informações sobre variações de temperatura na pele podem ser empregadas para a localização de regiões que contém células cancerígenas e, desta forma, propiciar um tratamento mais eficiente e menos agressivo para os pacientes.

Do ponto de vista prático, a localização de tumores se dá por meio da formulação e resolução de um problema inverso. Este consiste da definição da função objetivo, isto é, a partir do uso de informações sobre o perfil de temperatura e do emprego de um dado método de otimização, minimizar um determinado funcional. A determinação do perfil de temperatura em tecidos se dá por meio da modelagem da transferência de calor em tecidos. Esta caracteriza-se por ser um fenômeno complexo por envolver múltiplos mecanismos fenomenológicos, dentre os quais pode-se citar: condução, convecção, radiação, metabolismo, evaporação e mudança de fase. Além disso, há de se considerar que as propriedades térmicas dos tecidos são dependentes da temperatura e do teor de água. Outro aspecto relevante para a formulação do problema inverso é a capacidade do sistema termo-regulatório humano manter a temperatura média interna em torno de $37^{\circ} \mathrm{C}$ sob uma ampla faixa de condições ambientais externas e durante tensões térmicas (Pennes, 1948). Neste caso, uma pequena diferença de temperatura na pele pode implicar na presença de células cancerígenas que, a partir da formulação e resolução de um problema inverso, pode auxliar na identificação das reigões com células cancerígenas, possibilitando um tratamento mais eficaz. É importante ressaltar que a determinação de tais regiões é de suma importância, visto que esta identificação pode auxiliar os tratamentos tradicionais de modo a evitar ou minimizar prováveis danos térmicos à região sadia circunvizinha, para os casos onde o tratamento cirúrgico não é recomendável ou possível (Lima et al., 2006).

Diante do que foi apresentado, este trabalho tem por objetivo propor e resolver um problema inverso de transferência de calor em tecidos. Para essa finalidade, são empregados o Método das Diferenças Finitas (para resolver o problema direto) e o algoritmo de Evolução Diferencial (para resolver o problema inverso). A função objetivo é definida a partir de imagens de carcinomas de pele extraídas da literatura especializada. Este trabalho é estruturado conforme segue. A Seção 2 apresenta aspectos gerais sobre o câncer de pele, ressaltando os tipos mais comuns e a forma de tratamento. As Seções 3 e 4 apresentam a modelagem matemática do problema de interesse, da resolução do problema direto, uma breve descrição do algoritmo de Evolução Diferencial e a formulação do problema inverso. Na Seção 5 são apresentados os resultados obtidos com a aplicação em dois estudos de caso. Finalmente, as conclusões e perspectivas para trabalhos futuros são descritas na última seção.

\section{CÂNCER DE PELE}

Nos últimos anos, a incidência de câncer de pele no Brasil tem aumentado significativamente. Como acontece em outros tipos de carcinomas, se este for detectado no início, isto é, antes de atingir as camadas mais profundas da pele, a probabilidade de cura aumenta significativamente. Neste contexto, os seguintes aspectos podem indicar a presença de célular cancerígenas: $i$ ) observar os locais com a presença de pintas ou manchas, principalmente se estas forem recentes; ii) reparar se alguma destas manchas coçam, ardem ou sangram; iii) observar se uma ou mais destas mudaram de cor, de tamanho ou de textura; $i v$ ) ficar atento com lesões endurecidas ou que alteram sua tonalidade, $v$ ) observar se alguma ferida não se cicatriza. Além disso, pode-se observar se alguma marca (mancha ou pinta) se encaixa no ABCDE de risco para melanoma: A (Assimetria da lesão. Pintas simétricas costumam ser benignas, ao contrário das assimétricas), B (Bordas irregulares, cheias de reentrâncias e saliências, $\mathbf{C}$ (Cor. Se a pinta tiver dois ou mais tons, pode ser perigosa), D (Diâmetro. Pintas medindo mais que $6 \mathrm{~mm}$, são mais preocupantes) e E (Expansão, ou crescimento da lesão).

\subsection{Tipos de Câncer de Pele}

De forma geral, são três os tipos mais frequentes de câncer de pele, que se originam de diferentes células que compõem pele ${ }^{4}$.

- Basocelular: originado das células da camada basal, é o tipo de câncer de pele mais frequente (cerca de $70 \%$ dos casos) e apresenta menor potencial de malignidade. Atinge principamente pessoas de pele clara e está diretamente relacionado com a exposição solar acumulada ao longo da vida. Seu crescimento é lento e, muito raramente, se dissemina à distância (metástases), mas pode destruir os tecidos à sua volta, atingindo até cartilagens e ossos. Manifesta-se de várias maneiras, dentre as quais pode-se citar: feridas que não cicatrizam-se ou lesões que sangram com facilidade devido a pequenos traumatismos, conforme apresentado na Fig. 1.

\footnotetext{
${ }^{3}$ http://www.oncomedbh.com.br/

${ }^{4}$ http://www.sbd.org.br/
} 


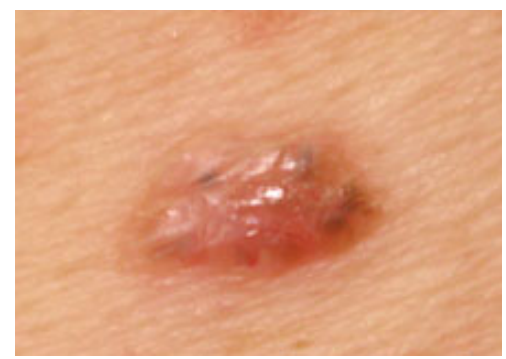

Figura 1. Figura de um carcinoma do tipo basocelular (Reproduzido de http://www.sbd.org.br/).

- Espinocelular: tem origens nas células da camada espinhosa, apresenta crescimento mais rápido do que o basoceluar, podendo enviar metástases à distância para gânglios linfáticos e outros órgãos. É o segundo tipo mais comum de câncer de pele, sendo bem menos frequente que o basocelular. Este carcinoma surge em áreas da pele que sofrem exposição prolongada ao sol e a partir de ceratoses actínicas (lesões decorrentes da exposição solar acumulada durante a vida e consideradas pré-cancerosas). Também é comum acometer áreas de mucosa aparente, como a boca ou o lábio (relacionados com o tabagismo), cicatrizes de queimaduras antigas, áreas da pele com processos inflamatórios crônicos, como as úlceras de perna, ou áreas que sofreram irradiação (raios X), conforme apresentado na Fig. 2.

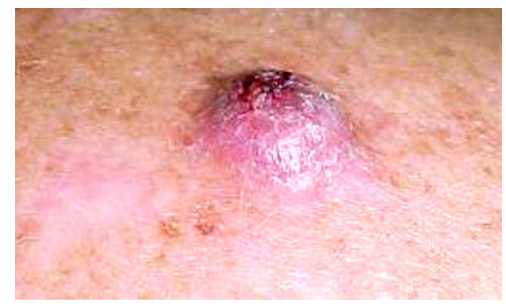

Figura 2. Figura de um carcinoma do tipo espinocelular (Reproduzido de http://www.sbd.org.br/).

- Melanoma: originado das células que produzem o pigmento da pele (melanócitos), é o câncer de pele mais perigoso. Isto se deve a frequência com que envia metástases para outros órgãos e que podem levar o paciente ao óbito. Este tipo de carcinoma pode surgir a partir da pele sadia ou a partir de regiões com pigmentação diferenciada. Apesar de ser mais frequente nas áreas da pele comumente expostas ao sol, o melanoma também pode ocorrer em áreas de pele não expostas. A Fig. 3 ilustra a forma base de um câncer do tipo melanoma.

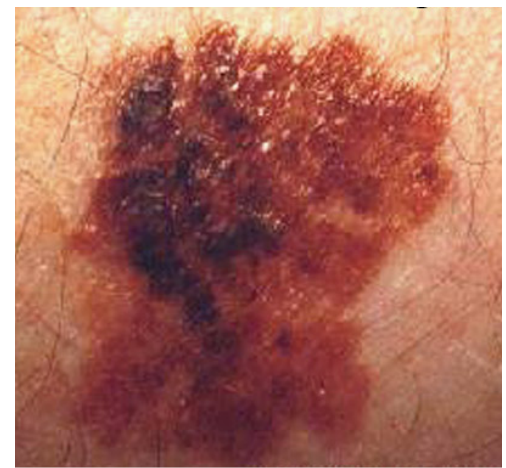

Figura 3. Figura de um carcinoma do tipo melanoma (Reproduzido de http://www.sbd.org.br/).

\subsection{Tipos de Tratamento para o Câncer de Pele}

Basicamente, o tratamento do carcinomas de pele restringem-se a procedimentos cirúrgicos, da destruição das lesões por radioterapia ou criocirurgia com nitrogênio líquido Agnelli et al. (2011). Independentemente da estratégia adotada, é importante ressaltar que a eficâcia do tratamento depende da precocidade da identificação do câncer, isto é, quanto antes a lesão for identificada e o tratamento implementado, maior é a chance de obter a cura, evitando desta forma a disseminação de células cancerígenas outros órgãos (metástases).

\section{FORMULAÇÃO MATEMÁTICA DO PROBLEMA DE BIO-TRANSFERÊNCIA DE CALOR EM TECIDOS}

Matematicamente, o processo de transferência de calor em tecidos pode ser modelado pela equação de Pennes (1948). Esta equação descreve o mecanismo de perfusão sanguínea no balanço de energia em tecidos. Em estado estacionário, a 
equação de Pennes é dada como segue (Agnelli et al., 2011):

$$
\lambda_{e} \nabla^{2} T_{e}+k_{e}\left(T_{b}-T_{e}\right)+Q_{m e}=0
$$

em que o subscrito $e=1,2$ identifica os subdomínios de tecido sadio e com tumor, respectivamente, $\lambda_{e}$ é a condutividade térmica, $k_{e}=G_{b e} c_{b}$ é o coeficiente de perfusão $\left(G_{b e}\right.$ é a taxa de perfusão sanguínea e $c_{b}$ é taxa volumétrica específica do sangue), $Q_{m e}$ é o termo fonte metabólico, $T_{b}$ é a temperatura do sangue e $T_{e}$ é a temperatura do tecido onde $e=1,2$ identifica os subdomínios de tecido sadio e com tumor, respectivamente. O primeiro termo do lado esquerdo da equação acima representa a condução global de calor. Já o segundo termo representa a transferência de calor convectiva que ocorre devida a diferença de temperatura entre o sangue e o tecido. O último termo representa o calor gerado por unidade de volume. As condições de contorno associados ao problema estudado são descritas a seguir (Agnelli et al., 2011):

$$
\begin{gathered}
\left.T_{1}\right|_{y=0}=T_{b} \\
-\left.\lambda_{1} \frac{\partial T_{1}}{\partial x}\right|_{x=0}=0 \\
-\left.\lambda_{1} \frac{\partial T_{1}}{\partial x}\right|_{x=x^{\max }}=0
\end{gathered}
$$

onde $\lambda_{1}$ é a condutividade térmica do tecido sadio.

Finalmente, na superfície da pele, a seguinte condição de convecção é imposta:

$$
-\left.\lambda_{1} \frac{\partial T_{1}}{\partial y}\right|_{y=y^{\max }}=\alpha\left(T_{1}-T_{a}\right)
$$

onde $\alpha$ é o coeficiente de transferência de calor e $T_{a}$ é a temperatura ambiente.

\subsection{Resolução do Problema Direto}

Para resolver este problema (direto) é empregado o Método das Diferenças Finitas com malhas igualmente espaçadas. Assim, o Laplaciano é aproximado por um esquema de segunda ordem $\left(\Delta x=\Delta y=h\right.$, na direção $x\left(i=1, \ldots, n_{x}\right)$ e na direção $y\left(j=1, \ldots, n_{y}\right)$, em que $h$ é o tamanho do passo usado para resolver a equação diferencial parcial descrita. Neste caso, a Eq. 1 pode ser reescrita como (Fortuna , 2000):

$$
\lambda_{i, j}\left(\left.\frac{\partial^{2} T}{\partial x^{2}}\right|_{i, j}+\left.\frac{\partial^{2} T}{\partial y^{2}}\right|_{i, j}\right)+k_{i, j}\left(T_{b}-T_{i, j}\right)+Q_{i, j}=0
$$

onde

$$
\begin{aligned}
& \left.\frac{\partial^{2} T}{\partial x^{2}}\right|_{i, j}=\frac{T_{i+1, j}-2 T_{i, j}+T_{i-1, j}}{h^{2}} \\
& \left.\frac{\partial^{2} T}{\partial y^{2}}\right|_{i, j}=\frac{T_{i, j+1}-2 T_{i, j}+T_{i, j-1}}{h^{2}}
\end{aligned}
$$

Similarmente, as condições de contorno também podem ser aproximadas.

$$
\begin{gathered}
T_{i, 1}=T_{b}, i=2, \ldots, n_{x}-1 \\
\left.\frac{\partial T_{1}}{\partial y}\right|_{1, j}=\frac{T_{2, j}-T_{1, j}}{h}=0, j=2, \ldots, n_{y}-1 \\
\left.\frac{\partial T_{1}}{\partial y}\right|_{n_{x}, j}=\frac{T_{n_{x}, j}-T_{n_{x}-1, j}}{h}=0, j=2, \ldots, n_{y}-1 \\
-\lambda_{i, j}\left(\frac{T_{i, n_{y}}-T_{i, n_{y}-1}}{h}\right)=\alpha\left(T_{i, n_{y}}-T_{a}\right)
\end{gathered}
$$

O sistema algébrico formado pelas Eqs. 6 à 12 é então resolvido utilizando o Método de Gauss-Siedel (Barroso et al., 1987).

\section{ALGORITMO DE EVOLUÇÃO DIFERENCIAL}

O algoritmo de Evolução Diferencial (ED) baseia-se na realização de operações vetoriais na qual a diferença ponderada entre dois indivíduos distintos, adicionada a um terceiro indivíduo, é o responsável pela geração de novos candidatos a solução do problema de otimização. O ED apresenta as seguintes operações: inicialização da população, mutação, cruzamento, seleção, e critério de parada. A seguir são descritas cada uma destas operações. 


\subsection{Inicialização}

O processo de inicialização consiste na geração de indivíduos de forma aleatória. Neste caso, de posse do número de indivíduos da população e do domínio de cada variável de projeto, geram-se números aleatórios que serão aplicados a esse intervalo, obtendo-se assim um vetor de indivíduos da população, como mostrado a seguir:

$$
x_{i, j}=x_{i, L}+\operatorname{rand}\left(x_{i, U}-x_{i, L}\right)
$$

onde $x_{i, L}$ e $x_{i, U}$ são os limites inferiores e superiores das $j$-ésimas variáveis de projeto, respectivamente, e rand é um gerador de números aleatórios entre 0 e 1 .

\subsection{Operador de Mutação}

Neste operador, o algoritmo de ED realiza as operações de mutação e recombinação para a geração de uma nova população com NP indivíduos. Este operador adiciona a um vetor de referência, escolhido aleatoriamente na população, um vetor diferença obtido a partir de outros dois vetores também escolhidos aleatoriamente na população, como descrito a seguir:

$$
v_{i, g}=x_{r 0, g}+F\left(x_{r 1, g}-x_{r 2, g}\right)
$$

onde $v_{i, g}$ é um novo vetor (candidato), o escalar $F$ (taxa de perturbação) é um número real que controla a magnitude do vetor diferença obtido em cada operação, e os vetores $x_{r 0, g}, x_{r 1, g}$ e $x_{r 2, g}$ são escolhidos aleatoriamente na população, como ilustrado na Tabela 1.

Tabela 1. Mecanismos de mutação no algoritmo de ED.

\begin{tabular}{ccc}
\hline Estratégia & Representação & Mecanismo \\
\hline 1 & $x^{j+1}=x_{b e s t}^{j}+F\left(x_{\kappa_{1}}^{j}-x_{\kappa_{2}}^{j}\right)$ & DE/BEST/1/EXP \\
\hline 2 & $x^{j+1}=x_{\kappa_{3}}^{j}+F\left(x_{\kappa_{1}}^{j}-x_{\kappa_{2}}^{j}\right)$ & DE/RAND/1/EXP \\
\hline 3 & $x^{j+1}=x_{o l d}^{j}+F\left(x_{b e s t}^{j}-x_{o l d}^{j}\right)+F\left(x_{\kappa_{1}}^{j}-x_{\kappa_{2}}^{j}\right)$ & DE/RAN-TO-BEST/2/EXP \\
\hline 4 & $x^{j+1}=x_{b e s t}^{j}+F\left(x_{\kappa_{1}}^{j}-x_{\kappa_{2}}^{j}\right)+F\left(x_{\kappa_{3}}^{j}-x_{\kappa_{4}}^{j}\right)$ & DE/BEST/2/EXP \\
\hline 5 & $x^{j+1}=x_{\kappa_{5}}^{j}+F\left(x_{\kappa_{1}}^{j}-x_{\kappa_{2}}^{j}\right)+F\left(x_{\kappa_{3}}^{j}-x_{\kappa_{4}}^{j}\right)$ & DE/RAND/2/EXP \\
\hline 6 & $x^{j+1}=x_{b e s t}^{j}+F\left(x_{\kappa_{1}}^{j}-x_{\kappa_{2}}^{j}\right)$ & DE/BEST/1/BIN \\
\hline 7 & $x^{j+1}=x_{\kappa_{3}}^{j}+F\left(x_{\kappa_{1}}^{j}-x_{\kappa_{2}}^{j}\right)$ & DE/RAND/1/BIN \\
\hline 8 & $x^{j+1}=x_{o l d}^{j}+F\left(x_{b e s t}^{j}-x_{o l d}^{j}\right)+F\left(x_{\kappa_{1}}^{j}-x_{\kappa_{2}}^{j}\right)$ & DE/RAND-TO-BEST/2/BIN \\
\hline 9 & $x^{j+1}=x_{b e s t}^{j}+F\left(x_{\kappa_{1}}^{j}-x_{\kappa_{2}}^{j}\right)+F\left(x_{\kappa_{3}}^{j}-x_{\kappa_{4}}^{j}\right)$ & DE/BEST/2/BIN \\
\hline 10 & $x^{j+1}=x_{\kappa_{5}}^{j}+F\left(x_{\kappa_{1}}^{j}-x_{\kappa_{2}}^{j}\right)+F\left(x_{\kappa_{3}}^{j}-x_{\kappa_{4}}^{j}\right)$ & DE/RAND/2/BIN \\
\hline
\end{tabular}

A convenção utilizada nesta tabela é $\mathrm{DE} / \mathrm{X} / \mathrm{Y} / \mathrm{Z}$, onde $\mathrm{X}$ representa o vetor que será perturbado (BEST ou RAND). Y é o número de pares de vetores que são considerados durante a perturbação e $\mathrm{Z}$ é o tipo de cruzamento usado para a geração do candidato, BIN no caso binomial e EXP no caso exponencial. Os subscritos $\kappa_{i}(i=1, \ldots ., 5)$ são índices escolhidos aleatoriamente na população. $x_{\text {best }}$ é o melhor indivíduo da população na geração anterior e $x_{o l d}$ é um indivíduo escolhido aleatoriamente dentro da população na geração anterior.

\subsection{Operador de Cruzamento}

Neste operador, o vetor $v_{i, g}$ gerado anteriormente pode ou não ser aceito na próxima geração de acordo com a seguinte condição:

$$
u_{i, g}= \begin{cases}v_{i, g} & \text { se } \text { rand } \leq C R \text { ou } j=j_{\text {rand }} \\ x_{i, g} & \text { caso contrário }\end{cases}
$$

onde $C R$ é denominada probabilidade de cruzamento, definida pelo usuário e pertencente ao intervalo [0, 1]. De forma geral, tal parâmetro controla as informações dos pais que serão transmitidas aos filhos. Para determinar qual a contribuição de um determinado vetor gerado, o cruzamento compara $C R$ com o gerador de números aleatórios rand. Se o número aleatório gerado é menor ou igual a $C R$, o vetor $v_{i, g}$ (com posição $j_{\text {rand }}$ ) é aceito, caso contrário, o vetor $x_{i, g}$ é mantido na população atual.

\subsection{Operador de Seleção}

Se o vetor $u_{i, g}$ tem melhor valor de função objetivo $(f)$ em relação ao vetor $x_{i, g}$, ele o substitui na próxima geração; caso contrário, $x_{i, g}$ é mantido na população por mais uma geração, como mostrado a seguir:

$$
x_{i, g+1}= \begin{cases}u_{i, g} & \text { se } f\left(u_{i, g}\right) \leq f\left(x_{i, g}\right) \\ x_{i, g} & \text { caso contrário }\end{cases}
$$

Finalmente, uma vez completado o processo de atualização da população, este processo é repetido até que um determinado critério de parada seja satisfeito. Neste capítulo, o critério de parada adotado foi o número máximo de gerações. 
Com relação à escolha dos parâmetros do algoritmo de ED, Storn e Price (1995) aconselham o uso dos seguintes valores: número de indivíduos da população como sendo igual a um valor entre 5 e 10 vezes o número de variáveis de projeto, taxa de perturbação $F$ entre 0,2 e 2,0, probabilidade de cruzamento $C R$ entre 0,1 e 1,0, e estratégia DE/RAND/1/BIN (Babu et al., 2005). É importante ressaltar que outros valores para esses parâmetros podem ser atribuídos de acordo com uma aplicação particular.

Na literatura especializada, inúmeras aplicações usando o algoritmo de ED podem ser encontradas, dentre as quais pode-se citar a determinação do perfil ótimo de alimentação de substrato em fermentadores (Kapadi e Gudi, 2004); a determinação das condições operacionais de um reator industrial utilizado para a produção de estireno (Babu et al., 2005); a otimização multi-objetivo de vigas (Lobato e Steffen Jr, 2007), a determinação do perfil ótimo de alimentação de substrato em um problema de controle ótimo com índice flutuante (Lobato et al., 2007), a estimação de parâmetros cinéticos em um secador rotatório (Lobato et al., 2008); o uso de técnicas de meta-modelagem associada ao enfoque multi-objetivo para o tratamento de um problema de interação fluido-estrutura (Lobato, 2008), o uso de técnicas de meta-modelagem aplicado ao processo de usinagem (Lobato et al., 2012a), estimação de propriedades em problemas de transferência de calor por radiação (Lobato et al., 2012b), estimação de parâmetros em pirólise (Santos et al., 2012), projeto de hidrociclones (Silva et al., 2012), entre outras aplicações.

\subsection{Formulação do Problema Inverso}

O problema inverso de tranferência de calor considerado neste trabalho consiste na estimativa da região $\Omega_{1}$ (definida pelo contorno $\Gamma$ ) que contém o tumor, de modo que $\Omega_{2}+\Omega_{1}=\Omega$ (ver Fig. 4), por meio da minimização do funcional $Q$, definido como sendo a diferença entre a temperatura experimental $\left(T^{e x p}\right)$ e calculada pelo modelo matemático $\left(T^{c a l}\right)$ :

$$
Q_{\Gamma}=\sum_{i=1}^{N} \sum_{j=1}^{M}\left(T_{i j}^{c a l}-T_{i j}^{\mathrm{exp}}\right)^{2}
$$

onde $N \times M$ é o número de dados experimentais considerados.

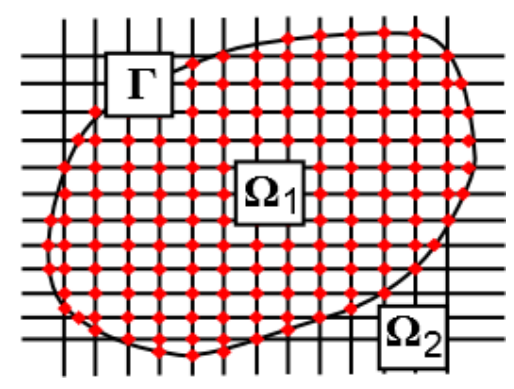

Figura 4. Domínio do problema de interesse (a região $\Omega_{1}$ contém as células canceerígenas e a região $\Omega_{2}$ contém apenas células sadias).

Para a caracterização das duas regiões (sadia e doente), define-se diferentes parâmetros ( $\lambda, k$ e $\left.Q_{m}\right)$ para cada uma delas de modo que o perfil de temperatura na região que contém células cancerígenas apresente um pequeno incremento de temperatura em relação a região sadia. Como dados experimentais reais para a temperatura não estão disponíveis, serão gerados dados experimentais sintéticos, computados a partir da seguinte equação:

$$
T_{i j}^{\mathrm{exp}}=T_{i j}^{c a l}+\kappa \xi
$$

onde $T_{i j}^{c a l}$ representa o perfil de temperatura conhecendo-se a região que contém as células cancerígenas, $\kappa$ é o desvio padrão dos erros de medida e $\xi$ é um número pseudo-aleatório que pertence ao intervalo de $[-1,1]$ (necessário para inserir a aleatoriedade ao processo de geração de dados experimentais sintéticos).

É importante ressaltar que a região que apresenta as células cancerígenas $\left(\Omega_{1}\right)$ é delimitada pelo contorno $\Gamma$ (ver Fig. 4). Este é computado a partir do uso de splines cúbicas (Barroso et al., 1987) definidas por elementos de controle a partir da geração de candidatos obtidos com a aplicação do algoritmo de ED. Para cada região definida pelo algoritmo de otimização, resolve-se o problema direto e compara-se o perfil de temperatura simulado com o experimental sintético. Neste caso, objetiva-se minimizar o funcional definido pela Eq. 17 a partir da determinação do contorno $\Gamma$.

\section{RESULTADOS E DISCUSSÃO}

Para ilustrar a metodologia proposta neste trabalho são considerados dois estudos de caso, caracterizados por diferentes contornos $\Gamma$. Em todos os estudos de caso foram considerados os seguintes parâmetros (Agnelli et al., 2011): $\lambda_{1}=0,5(\mathrm{~W} / \mathrm{m} \mathrm{K}), \lambda_{2}=0,75(\mathrm{~W} / \mathrm{m} \mathrm{K}), k_{1}=1998,1\left(\mathrm{~W} / \mathrm{m}^{3} \mathrm{~K}\right), k_{2}=7992,4\left(\mathrm{~W} / \mathrm{m}^{3} \mathrm{~K}\right), Q_{m 1}=4200\left(\mathrm{~W} / \mathrm{m}^{3}\right), Q_{m 2}=42000$ $\left(\mathrm{W} / \mathrm{m}^{3}\right), T_{b}=37^{\circ} \mathrm{C}, T_{a}=25^{\circ} \mathrm{C}, \alpha=10\left(\mathrm{~W} / \mathrm{m}^{2} \mathrm{~K}\right)$ e $n_{x}=n_{y}=50$ pontos (selecionados a partir de testes de independência de malha). Os subscritos 1 e 2 representam os tecidos sadio e com doente, respectivamente. Para a determinação de cada 
contorno via aplicação de splines cúbicas são considerados 50 elementos de controle. Além disso, para os estudos de caso com ruído, foram gerados pontos cuja magnitude do erro não ultrapassa 7\%. Os parâmetros utilizados no algoritmo ED são os seguintes (Storn e Price, 1995): tamanho da população igual a 50, taxas de cruzamento e perturbação iguais a 0,8 , e estratégia DE/1/rand para gerar candidatos em potencial para a resolução do problema inverso. O critério de parada considerado foi o número máximo de gerações (500). Neste caso, para os parâmetros definidos são necessárias 25050 avaliações da função objetivo em cada uma das 10 execuções realizadas para a obtenção dos valores médios que serão apresentados (são consideradas sementes iniciais diferentes para cada execução do algoritmo). Para resolver o sistema linear obtido com a discretização, foi empregado o Método de Gauss-Siedel (Barroso et al., 1987) com condição inicial igual a $37^{\circ} \mathrm{C}$ (para todas as incógnitas).

\subsection{Estudo de Caso 1}

A Figura 5 apresenta o carcinoma definido pelo contorno $\Gamma$ considerado nesta aplicação.

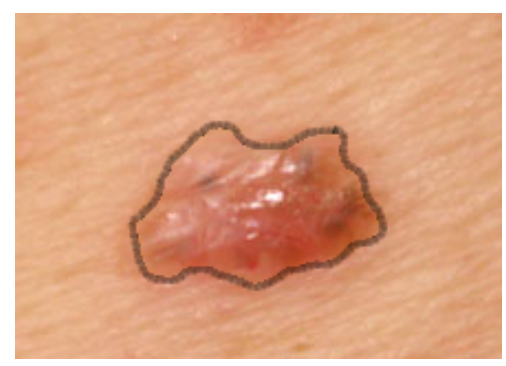

Figura 5. Contorno considerado no estudo de caso 1.

A Tab. 2 apresenta os resultados obtidos pelo algoritmo ED para o estudo de caso 1 considerando ou não a presença de ruídos nos dados experimentais.

Tabela 2. Resultados obtidos com o algoritmo de ED para o estudo de caso 1.

\begin{tabular}{|c|c|}
\hline Ruído (\%) & Função Objetivo (Eq. 17) \\
\hline 0 & $2,56 \times 10^{-6}\left(1,66 \times 10^{-8 \dagger}\right)$ \\
7 & $1,87 \times 10^{-2}\left(2,66 \times 10^{-3 \dagger}\right)$ \\
\hline
\end{tabular}

${ }^{\dagger}$ Desvio padrão obtido com as execuções do algoritmo de ED.

Nesta tabela é possível observar que a metodologia proposta foi capaz de estimar satisfatoriamente o contorno $\Gamma$, isto é, a região que contém as células cancerígenas através da formulação e resolução de um problema inverso utilizando o perfil de temperatura da pele. Como esperado, a introdução de ruído aos dados experimentais incrementa o valor da função objetivo definida. Todavia, mesmo com a magnitude do erro considerada, boas estimativas para o contorno foram obtidas.

\subsection{Estudo de Caso 2}

O último estudo de caso contempla o contorno do carcinoma definido na Fig. 6.

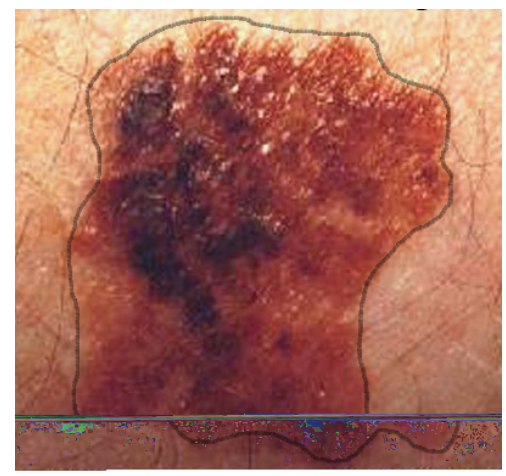

Figura 6. Contorno considerado no estudo de caso 2.

A Tab. 3 apresenta os resultados obtidos pelo algoritmo ED para o estudo de caso 2 considerando ou não a presença de ruídos nos dados experimentais. 
Tabela 3. Resultados obtidos com o algoritmo de ED para o estudo de caso 2.

\begin{tabular}{|c|c|}
\hline Ruído (\%) & Função Objetivo (Eq. 17) \\
\hline 0 & $3,44 \times 10^{-6}\left(3,87 \times 10^{-7 \dagger}\right)$ \\
7 & $2,33 \times 10^{-2}\left(5,67 \times 10^{-3 \dagger}\right)$ \\
\hline \multicolumn{2}{|c|}{${ }^{\dagger}$ Desvio padrão obtido com as execuções do algoritmo de ED. }
\end{tabular}

Conforme contatado na aplicação anterior, os resultados apresentados na Tab. 3 demonstram que a metodologia proposta foi capaz de estimar satisfatoriamente o contorno $\Gamma$. Além disso, como esperado, a introdução de ruído aos dados experimentais incrementa o valor da função objetivo definida. Todavia, apesar da magnitude do erro considerado, boas estimativas para o contorno foram obtidas.

\section{CONCLUSÕES}

No presente trabalho, o Algoritmo de Evolução de Diferencial associado com o Método das Diferenças Finitas, foi utilizado para determinar a região que contém células cancerígenas através da formulação e resolução de um problema inverso. Fisicamente, este problema foi modelado pela equação de transferência de calor em tecidos. Neste cenário, foram apresentados dois estudos de caso considerando imagens de carcinomas de pele extraídos da literatura especializada. De maneira geral foi possível concluir que a metodologia proposta conseguiu obter resultados satisfatórios para ambos os casos. Todavia, é importante ressaltar, apesar do problema sintético gerado, que a presente metodologia pode ser facilmente aplicada para problemas com dados experimentais reais obtidos a partir de termografia. Como trabalho futuro pretende-se analisar a presença de incerteza (otimização robusta) no procedimento de estimativa das variáveis, além de utilizar dados experimentais obtidos via termografia. Além disso, tem-se como objetivo secundário o estudo de outras técnicas para a resolução do problema direto formulado, dentre as quais pode-se citar o uso de métodos sem malha.

\section{AGRADECIMENTOS}

Os autores agradecem a FAPEMIG e ao CNPq pelo apoio financeiro para a participação no congresso e pelas bolsas de iniciação científica.

\section{REFERÊNCIAS}

Agnelli, J. P., Barrea, A., Turner, C. V., 2011. Tumor Location and Parameter Estimation by Thermography. Mathematical and Computer Modelling, vol. 53 (7-8), pp. 1527-1534.

Babu, B. V., Chakole, P. G., Mubeen, J. H. S., 2005. Multiobjective differential evolution (mode) for optimization of adiabatic styrene reactor. Chemical Engineering Science, vol. 60 (12), pp. 4822-4837.

Barroso, L. C., Barroso, M. M. A., Campos Filho, F. F., Carvalho, M. L. B., Maia, M. L., 1987. Cálculo Numérico com Aplicações. 2 Edição Harbra.

Fortuna, A. O., 2000. Técnicas Computacionais para a Dinâmica dos Fluidos. Editora da Universidade de São Paulo.

Kapadi, M. D., Gudi, R. D., 2004. Optimal control of fed-batch fermentation involving multiple feeds using differential evolution. Process Biochemistry, vol. 39 (3), pp. 1709-1721.

Lima, R. C. F., Lyar, P. R. M., Guimarães, C. S. C., Carvalho, D. K. E., Silva, G. M. L. L., 2006. Modelagem da biotransferência de calor no tratamento por hipertermia de tumores pelo MVF. Revista Brasileira de Engenharia Biomédica, vol. 22 (2), pp. 119-129.

Lobato, F. S., Steffen Jr, V., 2007. Engineering System Design with Multi-objective Differential Evolution. Proceedings in $19^{\text {th }}$ International Congress of Mechanical Engineering - Cobem 2007, Brasília, Brazil, pp. 1-10.

Lobato, F. S., Oliveira-Lopes, L. C., Murata, V. V., Steffen Jr, V., 2007. Solution of Multi-Objective Optimal Control Problems with Index Fluctuation using Differential Evolution. Proceedings in $6^{\text {th }}$ Brazilian Conference on Dynamics, Control and Applications - DINCON, 2007, São José do Rio Preto - SP., pp. 1-8.

Lobato, F. S., Arruda, E. B., Barrozo, M. A. S., Steffen Jr, V., 2008. Estimation of drying parameters in rotary dryers using differential evolution. Journal of Physics Conference Series, vol. 1, pp. 1-8.

Lobato, F. S., 2008. Otimização Multi-objetivo para o Projeto de Sistemas de Engenharia. Faculdade de Engenharia Mecânica.

Lobato, F. S., Malagoni, R. A., Souza, M. N., 2012. Differential Evolution Algorithm and Response Surface Methodology Applied to Turning Process Optimization. Materials Science Forum, vol. 727, pp. 1854-1859.

Lobato, F.S., Steffen Jr, V., Neto, A.J. Silva, 2012. Estimation of space-dependent single scattering albedo in a radiative transfer problem using differential evolution. Inverse Problems in Science \& Engineering, vol. 2, pp. 1-13.

Pennes, H. H., 1948. Analysis of Tissue and Arterial Blood Temperatures in the Resting Human Forearm. Journal of Applied Physiology, pp. 93-122.

Santos, K. G., Lobato, F. S., Barrozo, M. A. S., Murata, V. V., Lira, T. S., Gianesella, M. Bagasse Pyrolysis: A Comparative Study on Kinetic Models. Chemical Engineering Communications, vol. 199, pp. 109-121. 
Silva, D. O., Vieira, L. G. M, Barrozo, M. A. S., Lobato, F. S., 2012. Optimization of the Design and Performance of Hydrocyclones by Differential Evolution Technique. Chemical Engineering and Processing, vol. 61, pp. 1-7.

Storn, R., Price, K., 1995. Differential evolution: a simple and efficient adaptive scheme for global optimization over continuous spaces. International Computer Science Institute, vol. 12 (1), pp. 1-16.

\title{
8. RESPONSABILIDADE AUTORAL
}

Os autores são os únicos responsáveis pelo conteúdo deste trabalho.

\section{DEVELOPMENT OF A METHODOLOGY FOR THE CARCINOMAS TREATMENT THROUGH OF INVERSE PROBLEMS SOLUTION}

Fran Sérgio Lobato, fslobato@feq. ufu .br ${ }^{3}$

${ }^{1}$ Faculdade de Engenharia Química, Universidade Federal de Uberlândia, Brasil Av. João Naves de Ávila, 2.121 - Bloco 1K, 38408-100, Uberlândia - MG

\begin{abstract}
The engineering problems analysis through the formulation and solution of direct and inverse problems is an area with various applications. This interest is due to increase of realistic mathematical models associated with sophisticated numerical methods used for simulation. In this context, the application of inverse analysis to determine regions that presented cancerous cells is a task of great importance nowadays. In this contribution, the Differential Evolution Algorithm is used to determine regions with skin carcinomas by formulating and solving of an inverse problem through the heat transfer equation in tissues. The results obtained indicate that the proposed methodology is configured with as an interesting alternative for solve this inverse problem.
\end{abstract}

Keywords: Heat Transfer in Tissues, Inverse Problem, Differential Evolution. 